\title{
Correct Coding in Interventional Pain Management
}

\author{
Laxmaiah Manchikanti, MD
}

The National Correct Coding Council (NCCC) was created by the Health Care Financing Administration (HCFA) in 1996 to help ensure that providers across various jurisdictions receive like payment for the same services, use the same codes and provide similar documentation for services performed. The Correct Coding Initiative (CCI) was a direct outgrowth of the NCCC's, the purpose of which was to identify and isolate inappropriate coding, unbundling, and other irregularities in coding.

To avoid inappropriate or incorrect coding and billing in interventional pain management, it is imperative that interventional pain physicians and their staff be familiar with correct coding policies, as well as understand the meaning of Current Procedural Terminology, along with comprehensive codes, component codes, and mutually exclusive codes.

This review describes CCI and various correct coding policies specifically relevant to interventional pain medicine. In addition, certain commonly used codes of interventional techniques are also described with implications of comprehensive, component, and mutually exclusive coding terminology.

Keywords: National Correct Coding Policy, Correct Coding Initiative, Correct Coding Edits, comprehensive codes, component codes, mutually exclusive codes, interventional pain medicine
It is impossible to discuss interventional pain medicine without mentioning correct coding, billing, appropriate documentation, and fraud and abuse. Never in US history has there been such an emphasis on the description and definition of what the physician does for and to the patient. Compliance with the laws and regulations encompassing documentation with coding, billing, and collections, along with documentation, and medical records, is of crucial importance in today's interventional pain medicine practices. While the Balanced Budget Act of 1997 empowered and directed the Health Care Financing Administration (HCFA) to develop a payment system based upon physician work, and guidelines to document that work, the Kennedy-Kassebaum Health Reform Bill of 1996 provided the Office of Inspector General and the Federal Bureau of Investigations with broad powers and directed them to identify and prosecute health-care fraud and abuse. As such, it is the responsibility of HCFA to make certain that uniform payment policies and proce-

From Pain Management Center of Paducah, Paducah, Kentucky. Dr. Manchikanti is medical director of Pain Management Center of Paducah. Address correspondence: Laxmaiah Manchikanti, M.D., 2831 Lone Oak Road, Paducah, Kentucky 42003. Email: drm@apex.net dures are followed by all carriers, except in those instances where carriers are authorized to make local medical review policy. To help ensure that providers across various jurisdictions receive like payments for the same services (altered only by mandated geographic adjustments), use the same codes and provide similar documentation for services performed, HCFA created the National Correct Coding Council (NCCC). The purpose of the NCCC was to develop strategies for HCFA's Bureau of Program Operations to control improper coding that leads to inappropriate or increased payments in Part B claims. As a direct outgrowth of NCCC's work, HCFA established the National Correct Coding Policy in 1996 and eventually implemented the Medicare Correct Coding Initiative (CCI) to identify and isolate inappropriate coding, unbundling, and other irregularities in coding. Multiple versions of National Correct Coding Policies have been released in the form of a National Correct Coding manual ranging from version 5.0 to 6.2. In addition, HCFA also has utilized unpublished coding edits referred to as "black-box edits," which, essentially, are a system of payment denials to be used by carriers based on commercial utilization guidelines; carriers refuse to give beneficiaries and providers useful, complete information as to the rationale for these denials on the grounds that the guidelines used are "property" and, thus, confidential. Along with published 
CCIs, black-box edits are updated frequently and have become one of the most contentious issues related to Medicare and private insurance reimbursement in today's practice of medicine. Even though there have been a multitude of arguments on the issue of black-box edits and total elimination of these, it appears that at the present time, black-box edits are well and alive and growing rapidly, encroaching on all aspects of the practice of medicine.

The NCCC's policies are based on established coding conventions defined in the American Medical Association's (AMA's) Current Procedural Terminology (CPT) manual, national and local policies and edits, coding guidelines developed by national societies, analysis of standard medical and surgical practice, and reviews of current coding practices.

Correct coding essentially means reporting a group of procedures with appropriate comprehensive code. Under the CCI, HCFA has developed general policies that define the coding principles and edits that apply to procedure and service codes. Prior to development of CCI edits, Medicare Part B carriers included in their claims-processing systems various computerized edits to detect improper coding of procedures, which at the time was designated as fragmentation.

In consideration of monumental changes in outpatient coding with multiple proposals in 1999 and 2000 by HCFA, development of multiple new codes and revision of codes along with deletion of codes by AMA for CPT 2000 , and use of modifiers and ambulatory payment classifications by HCFA, HCFA officials have acknowledged that they have assigned over 105,000 coding edits to edit approximately 5,600 CPT codes Part B carriers use to analyze claims. These edits will be applied to physician services, services provided in ambulatory surgical centers, and hospital outpatient department claims.

\section{IMPACT OF CORRECT CODING POLICIES}

Since the installation of the Correct Coding Edits, which went into effect January 1, 1996, Medicare claims of inappropriate coding practices by providers and, thus, rejections have multiplied, resulting in substantial cost savings for the Medicare program. However, with the passage of the Health Insurance Portability and Accountability Act of 1996 (HR 3103), correct coding practices became part of the requirement to obtain proper payment from Medicare, rather than an option. The HCFA's current coding policies and edits apply when the same provider bills for all the procedures involved, or when the services for the same beneficiary were provided on the same day.

\section{GENERAL CORRECT CODING POLICIES}

In order for the CCI to be effective, it is essential that the coding description accurately describes what actually transpired at each patient encounter. A multitude of codes reflect the wide spectrum of services provided by various medical care providers, and many medical services can be rendered by different methods and combinations of various procedures. Hence, multiple codes describing similar services are frequently necessary to accurately reflect the particular service a physician performs. However, when multiple procedures are performed at the same session, the procedure and postprocedure work do not have to be repeated for each procedure; and, therefore, a comprehensive code describing the multiple services commonly performed together can be used. Many activities which are integral to a procedure are considered as generic activities and are assumed to be included as acceptable medical/surgical practice and, while they could be performed separately, they should not be considered as such when a code narrative is defined. Hence, all services integral to accomplishing a procedure will be considered to be included in that procedure and, therefore, will be considered a component and part of the comprehensive code. Various general correct coding policies are illustrated in Table $1(2,3)$.

Table 1. Various policies incorporated in correct coding policies

Standards of medical/surgical practice

Medical/surgical package

Services supplemental to a principal procedure (add - on codes)

CPT procedure code definition

CPT coding manual instructions or guidelines

Separate procedures

Designation of sex

Family of codes

Most extensive procedures

Sequential procedures

With or without procedures

Laboratory panels

Unlisted services or procedures

Misuse of column II code with column I code

Mutually exclusive procedures

Correct Coding Edits modifier indicator 


\section{Standards of Medical Surgical Practice}

Many of the provider activities during a procedure are integral to a procedure and termed as generic activities, which are assumed to be included as acceptable medical/ surgical practice, considered included in that procedure and considered a component of the procedure. Some generic services integral to standard medical/surgical services include:

- Cleansing, shaving, and prepping of the skin;

- Draping of the patient;

- Positioning of the patient;

- Insertion of intravenous access;

- Administration of sedation;

- Local, topical, or regional anesthetic administration;

- Identification the of surgical approach;

- Surgical cultures;

- Wound irrigation;

- Insertion and removal of drains, suction devices, dressings, and pumps;

- Application, management and removal of postoperative dressings, including transcutaneous electrical nerve stimulation units and institution of patient-controlled analgesia;

- Preoperative, intraoperative, and postoperative documentation; and

- Surgical supplies, unless excepted by existing HCFA policy.

\section{Medical/Surgical Package}

Over the years the CPT manual has grown to accommodate the expanding variety of surgical, diagnostic, and therapeutic surgical, as well as nonsurgical, procedures performed. In general, most services include associated preprocedure and postprocedure work; when performed at a single patient encounter, the preprocedure and postprocedure work is relatively fixed, regardless of the number of services actually performed at each session. For interventional pain procedures, some general guidelines can be developed. Thus, the following services are considered integral to an interventional procedure and are included in the CPT code description for the primary or comprehensive procedure. Such component services are:

- Intravenous access, e.g., CPT codes 36000, $36140,36400,36410,37201$, and 90780 to 90784;
- Cardiopulmonary monitoring, e.g., CPT codes 93000, 93005, 93040, 93041, 94656, 94760, 94761, or 94770 ;

Billing of successful service only;

Anesthesia by physician or conscious sedation, e.g., CPT codes 99141 and 99142.

\section{Add - on Codes}

The CPT coding system identifies certain codes that are submitted with other codes. These codes are identified generally with a statement such as, "List separately in addition to code for primary procedure in parentheses," but sometimes the supplemental code is to be used only with certain primary codes that are identified in parentheses. The purpose of these CPT codes is to enable providers to separately identify a service that is performed in certain situations as an additional service (3-5). Incidental services that are necessary to accomplish the primary procedure, such as injection of contrast, are not separately reported. Iatrogenic complications arising in the course of a procedure such as a catheter kink or malfunction requiring a replacement are not separately reported.

Add - on codes relevant to interventional pain management are: subsequent transforaminal epidural injections (CPT codes 64480 and 64484), facet-joint blocks (CPT codes 64472 and 64476), and facet-joint neurolysis (CPT codes 64623 and 64627).

\section{Modifiers}

In order to expand the information provided by the fivedigit CPT codes, a number of modifiers have been created by the AMA, HCFA, and local Medicare carriers. These modifiers, in the form of two digits, either numbers, letters, or a combination of each, are intended to convey specific information regarding the procedure or service to which they are appended (2). Modifiers are attached to the end of a code to indicate that a service or procedure described in the code definition has been modified by some circumstance. However, explicit understanding of the purpose of each modifier is required prior to its usage. It is also essential to recognize that modifiers may be different for each locality. In addition, it is essential to understand the specific meaning of the modifier for the payor to which a claim is being submitted before using it. For example, all modifiers described in the CPT code manual are not accepted by HCFA, third-party payors, Workmans Compensation carriers, and local carriers. Similarly, modifiers developed by HCFA or local Medi- 
care carriers are not accepted by third-party carriers or Workman's Compensation carriers. Meanwhile, Workman's Compensation carriers and third-party carriers also have developed their own modifiers in some jurisdictions.

Within the context of multiple-services reporting, without the addition of an appropriate modifier, it will appear that providers are engaging in the practice of "unbundling." The appropriate use of modifiers indicates that the services were performed under circumstances which did not involve this practice at all.

The HCFA identified modifier - 59 for use when several procedures are performed on different anatomic sites, or at different sessions on the same day (formerly known as the "GB" modifier) (3). This is considered as a distinct procedural service and the specific proposed language is: "Under certain circumstances, the physician may need to indicate that a procedure or service was distinct or independent from other services performed on the same day." Modifier - 59 is used to identify procedures/services that are not normally reported together, but are appropriate under the circumstances. However, modifier - 59 should be used only when no more descriptive modifiers such as an anatomic modifier or the staged-procedure modifier is available. Modifier - 50 is used to report bilateral procedures. Until April 1, 2000, HCFA's position was that facet-joint injections, neurolytic blocks and transforaminal injection codes were unilateral; however, now HCFA recognizes that these are bilateral codes.

\section{CPT Procedure Code Definition}

All procedures described by the CPT code narrative for the comprehensive code should have been performed, and only the single CPT code most accurately describing the procedure should be reported (1-3).

Thus, in interventional pain management, if a percutaneous lysis of adhesions is performed, CPT code 62263 with a description of the code, percutaneous lysis of epidural adhesions using solution injection, e.g., hypertonic saline, enzyme, or mechanical means, e.g., spring-guide catheter, including radiologic localization (includes contrast when administered) is used. It will be considered as unbundling, also fraud, if, in addition to 62263, other codes such as 62310 or 62311,62318 or 62319,62483 or $64484,72275,76005,62281$ or 62282 are used.

Similarly, facet-joint nerve blocks performed with neu- rolysis are considered as an integral part of the procedure. If epidural injection via indwelling catheter is utilized, separate codes for epidurography, or epidural injections are not advisable.

In addition, a code description may define a correct coding relationship where one code is a part of another based on the language used in the descriptor. Some examples of this type of correct coding in interventional pain medicine by code definition are: "single" and "multiple" codes - the single procedure is included in the multiple procedure, e.g., CPT 64420 intercostal nerve, single - CPT 64421 intercostal nerves, multiple.

Other examples, though not specific to interventional pain medicine, are:

"Partial" and "complete" CPT codes; partial is
included in complete
"Partial" and "total" CPT codes; partial is in-
cluded in total.
"Unilateral" and "bilateral" CPT codes; unilat-
eral procedure is included in bilateral.

\section{CPT Coding Manual Instruction Guidelines}

Each section of the CPT coding manual includes instructions that are unique to that section, in addition to the general instructions provided. These directions are not all inclusive of, nor limited to, definitions of terms, modifiers, unlisted procedures or services, special or written reports, details about reporting separate, multiple or starred procedures and qualifying circumstances $(2,4,5)$. These instructions define items or provide explanations that are necessary to appropriately interpret and report the procedures or services and to define terms that apply to a particular section.

Many changes have occurred in interventional pain procedure coding in the year 2000 CPT procedure manual; hence, it is imperative that an interventional pain physician thoroughly understand each procedure code used in describing interventional pain procedures to avoid misunderstanding, incorrect coding or unbundling.

\section{Separate Procedures}

The "separate procedure" notation in the CPT manual identifies a procedure or service that can be performed independently but that, when performed as an integral part of the comprehensive procedure, should not be re- 
ported separately. In previous CPT manuals, all epidural codes were considered as separate procedures; however, 2000 CPT codes with new epidural codes are not considered as separate procedures, since CPT code definitions have been expanded to clearly delineate what the CPT panel meant to include in that code without any ambiguity. Use of epidural codes, for example, in conjunction with percutaneous lysis of adhesions, will be a blatant fraud. However, the question remains as to whether an interlaminar epidural can be coded in conjunction with a transforaminal epidural, performed on occasions to reach two different areas.

\section{Designation of Sex}

Many procedure codes have a sex designation within their narrative; however, these codes do not apply to the practice of interventional pain medicine.

\section{Family of Codes}

The CPT manual describes certain codes that include two or more component codes that should not be reported separately, as these are considered members of a code family and included in a more comprehensive code. As such, comprehensive codes include certain services that are separately identified by other component codes. Although, component codes as members of the comprehensive code family represent parts of the procedure, that should not be listed separately when the complete procedure is performed, the component codes are considered individually if the procedures they describe are performed independently of the complete procedure. If this is not the case, all services listed in the comprehensive codes will be considered to make up the total service.

The example in interventional pain procedures would include epidurography, epidural catheterization, epidural steroid injection, epidural local anesthetic injection, injection of enzyme or neurolytic solution such as hypertonic saline or contrast, and needle localization for percutaneous lysis of adhesions, which are all component codes to describe the comprehensive procedure, namely, percutaneous lysis of adhesions.

Similarly, a comprehensive code is available describing epidural catheterization, hence, injection into the epidural space of local anesthetic steroid, contrast, etc., is not reportable separately.

A list of comprehensive and component codes relevant to the interventional pain specialist, with interventional pain procedures, appears in Tables 2 and 3.

\section{Most Extensive Procedures}

The HCFA-established policy is that, for a code which applies to a procedure that can be performed at different

Table 2. Correct coding edits for spinal and epidural injections based on CPT 2000 codes

\begin{tabular}{|c|c|c|}
\hline Comprehensive code & Component code(s) & $\begin{array}{l}\text { Mutually exclusive } \\
\text { code(s) }\end{array}$ \\
\hline 62270 - Lumbar - spinal puncture & $76000^{1}, 76001^{1}, 76003^{1}, 69990^{\circ}, 62273^{1}$ & None \\
\hline 62272 - Drainage of spinal fluid & $76000^{1}, 76001^{1}, 76003^{1}, 69990^{\circ}, 62270^{1}, 62273^{1}$ & None \\
\hline 62273 - Epidural blood patch & $\begin{array}{l}36000^{1}, 36140^{1}, 36410^{1}, 76000^{1}, 76001^{1}, 76003^{1}, 69990^{0} \text {, } \\
\mathrm{G}^{1} 001^{0}\end{array}$ & None \\
\hline 62280 - Neurolytic subarachnoid & $76000^{1}, 76001^{1}, 76003^{1}, 62270^{1}, 62272^{1}, 62273^{1}, 69990^{0}$ & None \\
\hline 62281 - Cervical epidural neurolytic & $76000^{1}, 76001^{1}, 76003^{1}, 62270^{1}, 62272^{1}, 62273^{1}, 69990^{0}$ & None \\
\hline 62282 - Lumbar epidural neurolytic & $76000^{1}, 76001^{1}, 76003^{1}, 62270^{1}, 62272^{1}, 62273^{1}, 69990^{0}$ & None \\
\hline 62310 - Cervical/thoracic epidural & $76001^{1}, 76003^{1}, 69990^{0}$ & None \\
\hline 62311 - Lumbar epidural & $76001^{1}, 76003^{1}, 69990^{\circ}$ & None \\
\hline 62318 - Cervical continuous epidural & $\begin{array}{l}76000^{1}, 76001^{1}, 76003^{1}, 36000^{1}, 36140^{1}, 36410^{1}, 62270^{1}, \\
62272^{1}, 62310^{1}, 69990^{\circ}\end{array}$ & $62273^{1}$ \\
\hline 62319 - Lumbar continuous epidural & $\begin{array}{l}76000^{1}, 76001^{1}, 76003^{1}, 36000^{1}, 36140^{1}, 36410^{1}, 62270^{1}, \\
62272^{1}, 63110^{1}, 20610^{1} 69990^{0}, 01996^{0}\end{array}$ & $62273^{1}$ \\
\hline 64479 - Cervical/thoracic transforaminal epidural, single & $76000^{1}, 76001^{1}, 76003^{1}, 69990^{0}$ & None \\
\hline level 6448 - Cervical/thoracic transforaminal, each additional & $69990^{\circ}$ & None \\
\hline level 648 - Lumbar/sacral transforaminal, single level & $76000^{1}, 76001^{1}, 76003^{1}$ & None \\
\hline
\end{tabular}


Table 3. Correct coding edits for facet-joint injections and neurolytic blocks, other nerve blocks, and injections, based on CPT 2000 codes

\begin{tabular}{|c|c|c|}
\hline Comprehensive code & Component code(s) & $\begin{array}{l}\text { Mutually } \\
\text { exclusive } \\
\text { codes }\end{array}$ \\
\hline $\begin{array}{l}64475 \text { - Lumbar/sacral facet-joint } \\
\text { block, single level }\end{array}$ & $76000^{1}, 76001^{1}, 76003^{1}, 69990^{\circ}$ & None \\
\hline $\begin{array}{l}64622 \text { - Lumbar or sacral facet } \\
\text { neurolysis, single level }\end{array}$ & $76000^{1}, 76001^{1}, 76003^{1}, 69990^{\circ}$ & None \\
\hline 27096 - Sacro-iliac joint injection & $76000^{1}, 76001^{1}, 76003^{1}, 69990^{\circ}$ & None \\
\hline $\begin{array}{l}62287 \text { - Percutaneous decompression } \\
\text { procedure of intervertebral disc }\end{array}$ & $62290^{1}, 76000^{1}, 76001^{1}, 76005^{1}, 69990^{\circ}$ & None \\
\hline 62290 - Lumbar discography & $69990^{\circ}$ & None \\
\hline 62291 Cervical discography & $69990^{\circ}$ & None \\
\hline $\begin{array}{l}62292 \text { - Injection procedure for } \\
\text { chemonucleolysis }\end{array}$ & $62290^{1}, 76000^{1}, 69990^{\circ}$ & None \\
\hline 64405 - Greater occipital nerve block & $69990^{\circ}$ & None \\
\hline 64420 - Intercostal nerve block, single & $69990^{\circ}$ & None \\
\hline $\begin{array}{l}64421 \text { - Intercostal nerve block, } \\
\text { multiple }\end{array}$ & $64420^{1}, 69990^{\circ}$ & None \\
\hline 64425 - Ilio-inguinal nerve block & $69990^{\circ}$ & None \\
\hline 64450 - Peripheral nerve block & $69990^{\circ}$ & None \\
\hline 64505 - Sphenopalatine ganglion & $69990^{\circ}$ & None \\
\hline b4sço - Stellate ganglion block & $69990^{\circ}$ & None \\
\hline $\begin{array}{l}64520 \text { - Lumbar or thoracic } \\
\text { sympathetic block }\end{array}$ & $69990^{\circ}$ & None \\
\hline 64530 - Celiac plexus block & $69990^{\circ}$ & None \\
\hline $\begin{array}{l}64600 \text { - Trigeminal neurolytic-small } \\
\text { branch }\end{array}$ & $64400^{1}, 69990^{0}$ & None \\
\hline $\begin{array}{l}64605 \text { - Trigeminal neurolytic- } 2 / 3 \\
\text { division }\end{array}$ & $69990^{\circ}$ & None \\
\hline $\begin{array}{l}64610 \text { - Trigeminal neurolytic-at } \\
\text { foramen ovale }\end{array}$ & $64605^{1}, 69990^{\circ}$ & None \\
\hline 64620 - Intercostal neurolytic & $64420^{1}, 64421^{1}, 69990^{0}$ & None \\
\hline 64640 - Peripheral neurolytic block & $\begin{array}{l}64405^{1}, 64408^{1}, 64410^{1}, 64412^{1}, 64413^{1}, 64415^{1}, 64417^{1}, 64418^{1}, 64425^{1} \\
, 64435^{1}, 64440^{1}, 64441^{1}, 64445^{1}, 64450,69990^{0}\end{array}$ & None \\
\hline $\begin{array}{l}20550 \text { - Injection, tendon sheath, } \\
\text { ligament, trigger points or ganglion } \\
\text { cyst }\end{array}$ & $\begin{array}{l}10160^{1}, 11900^{1}, 11901^{1}, 12032^{1}, 12042^{1}, 20500^{1}, 29075^{1}, 29105^{1}, 29125^{1}, \\
29130^{1}, 29220^{1}, 29260^{1}, 29405^{1}, 29425^{1}, 29450^{1}, 29515^{1}, 29530^{1}, 29540^{1}, \\
29550^{1}, 29580^{1}, 29590^{1}, 64405^{1}, 64442^{1}, 64445^{1}, 64450^{1}, 64550^{1}, 64717^{1}, \\
69990^{\circ}, 72240^{1}, 72265^{1}, 72295^{1}, 76000^{\circ}, 76003^{1}, 87102^{1}, 87163^{0}, 90780^{1}, \\
90781^{1}, 90782^{1}, 95900^{1}\end{array}$ & $11010^{1}$ \\
\hline $\begin{array}{l}20600 \text { - Arthrocentesis, aspiration } \\
\text { and/or injection; small joint, bursa or } \\
\text { ganglion cyst, e.g., fingers, toes }\end{array}$ & $\begin{array}{l}10060^{1}, 10061^{1}, 10140^{1}, 10160^{1}, 11719^{1}, 20500^{1}, 20550^{1}, 29065^{1}, 29075^{1}, \\
29085^{1}, 29105^{1}, 29125^{1}, 29130^{1}, 29260^{1}, 29280^{1}, 29365^{1}, 29405^{1}, 29425^{1}, \\
29505^{1}, 29515^{1}, 29540^{1}, 29550^{1}, 29580^{1}, 29590^{1}, 64450^{1}, 64704^{1}, 64708^{1}, \\
69990^{\circ}, 72240^{1}, 72265^{1}, 76000^{\circ}, 76003^{1}, 90780^{1}, 90782^{1}, 95900^{1}, G_{0127^{1}},\end{array}$ & $11010^{1}$ \\
\hline $\begin{array}{l}20605 \text { - Arthrocentesis, aspiration } \\
\text { and/or injections; intermediate joint, } \\
\text { bursa or ganglion cyst, e.g., } \\
\text { temporomandibular, } \\
\text { acromioclavicular, wrist, elbow or }\end{array}$ & $\begin{array}{l}10060^{1}, 10061^{1}, 10140^{1}, 10160^{1}, 11900^{1}, 12011^{1}, 15852^{1}, 20550^{1}, 29065^{1}, \\
29075^{1}, 29085^{1}, 29105^{1}, 29125^{1}, 29126^{1}, 29240^{1}, 29260^{1}, 29405^{1}, 29425^{1}, \\
29445^{1}, 29505^{1}, 29515^{1}, 29540^{1}, 29580^{1}, 29590^{1}, 29705^{1}, 64450^{1}, 64550^{1}, \\
64704^{1}, 69990^{0}, 76000^{\circ}, 76001^{1}, 76003^{1}, 90780^{1}, 90782^{1}, 95900^{1}\end{array}$ & $11010^{1}$ \\
\hline $\begin{array}{l}\text { 206le olecranon bursa } \\
\text { and/or injection; major joint or bursa, } \\
\text { e.g., shoulder, hip, knee joint, } \\
\text { subacromial bursa }\end{array}$ & $\begin{array}{l}10060^{1}, 10061^{1}, 10140^{1}, 10160^{1}, 11900^{1}, 12001^{1}, 12002^{1}, 12020^{1}, 12031^{1}, \\
12044^{1}, 15851^{1}, 20500^{1}, 20501^{1}, 20550^{1}, 29065^{1}, 29075^{1}, 29085^{1}, 29105^{1}, \\
29125^{1}, 29130^{1}, 29240^{1}, 29260^{1}, 29345^{1}, 29355^{1}, 29365^{1}, 29405^{1}, 29425^{1}, \\
29505^{1}, 29515^{1}, 29530^{1}, 29540^{1}, 29580^{1}, 64450^{1}, 64550^{1}, 64553^{1}, 64718^{1}, \\
69990^{0}, 72255^{1}, 72265^{1}, 72295^{1}, 76000^{0}, 76003^{1}, 76080^{1}, 90780^{1}, 90781^{1}, \\
90782^{1}, 95900^{1}\end{array}$ & $11010^{1}$ \\
\hline
\end{tabular}

levels of complexity, only the most extensive service, encompassing the comprehensive code actually performed, should be reported. Thus, when procedures are performed together that are basically the same, or performed on the 
same side but qualified by an increased level of complexity, the less extensive procedure is included in the more extensive procedure. Hence, the procedure viewed as the most complex should be reported.

\section{Sequential Procedures}

Sequential procedures or a successful procedure is identified in this policy. Which identifies codes for procedures that are often performed in sequence or for procedures involving an initial approach followed by a more invasive procedure during the same encounter. Only the procedure that successfully accomplishes the expected result is reported, with a less extensive procedure bundled into the more extensive one.

An example of this situation in interventional pain medicine would be a failed caudal epidural injection followed by percutaneous lysis of adhesions on the same day, in which case only the code for the successful procedure, that is, percutaneous lysis of adhesions, may be reported.

\section{With or Without Procedures}

Certain codes in the CPT manual identify a procedure that can be performed with or without certain services. It is contradictory to report code combinations in which one code represents a procedure that includes a certain service and the other code represents the procedure without that service.

As a practical matter in interventional pain management, multiple codes are described with or without contrast, or with or without radiological guidance.

\section{Laboratory Panels}

Medical necessity for laboratory evaluation must be established. In addition, an interventional pain specialist should pay close attention to comprehensive panel codes that include multiple component tests.

\section{Unlisted Services or Procedures}

Multiple sections in the CPT manual list certain codes that end in "99" or "9," in a few cases used to report a service that is not described in any code listed elsewhere in the CPT manual. This facilitates advances in technology, or physician expertise with new procedures when a CPT code may not have been assigned to a procedure when it is first introduced as accepted treatment. In these cases, at least initially, the unlisted service or procedure codes are necessary to describe the procedure. However, every effort should be made to find the appropriate code to describe the service, and frequent use of these unlisted codes instead of proper codes is not appropriate. Under this policy, the correct code would be assigned after the documentation has been reviewed, and then code pairs would be bundled based on this initial code. Thus far, the unlisted service or procedure has not been included in the CCI because of the multiple procedures that can be assigned to these codes.

It is interesting to note that, whenever a code is not available, well-meaning consultants advise to use an unlisted code; however, this may result not only in denial of payment for such a service, but also in raising a red flag if this code is repeatedly used.

The advice of manufacturers and the opinion of AMA has differed on multiple occasions regarding the description of spinal endoscopy, as well as electrothermal annuloplasty in interventional pain practices. A further disadvantage of using an unlisted code is that, even if reimbursement comes through for physician services, there will not be facility reimbursement for ambulatory surgery centers and hospital outpatient departments. Prior to development of CPT 2000 codes, many also have advised using unlisted codes to describe cervical facet-joint nerve blocks, cervical facet-joint neurolysis, and transforaminal injections.

\section{Misuse of Column II Code with Column 1 Code}

This policy indicates that CPT codes are written precisely and are not to be used out of context. At the same time, the policy also indicates that inappropriate interpretation of the CPT code definitions must not be performed. Thus, according to this policy, component codes cannot be billed with a comprehensive code.

Mutually exclusive codes are codes for procedures that cannot reasonably be performed in the same session.

\section{Correct Coding and its Modifier Indicator}

An appropriate modifier must be used with a code for which a modifier is appropriate. This code may be a column I or column II code. The definition of a modifier according to the CPT manual is as follows: "a modifier provides the means by which the reporting physician or provider can indicate that a service or procedure that has 
been performed has been altered by some specific circumstance but not changed in its definition or code."

Thus, the correct coding file formats have been presumably expanded to include a modified indicator for both the mutually exclusive code and the comprehensive component code tables. The Correct Coding Edits modifier indicators are " 0 ", "1", or "9." A " 0 " indicates that there are no circumstances in which a modifier would be appropriate. The services represented by the code combination will not be paid separately. A " 1 " indicates that a modifier is allowed in order to differentiate between the services provided. A " 9 " indicator is used for all code pays having a deletion date the same as their effective date.

\section{INCORRECT CODING}

Incorrect coding is defined as the intentional or unintentional billing of multiple procedure codes for a group of procedures that are covered by a single comprehensive code. Incorrect coding includes both unbundling and upcoding. Various types of incorrect coding examples include:

- Fragmenting one service into component parts and coding each component as if it were a separate service;

- Reporting separate codes for related services when the comprehensive code includes all related services;

- Breaking out bilateral procedures when one code is appropriate;

- Downcoding a service in order to use an additional code when one higher-level, more comprehensive code is appropriate; and

- Separating a surgical approach from a major surgical service.

Table 4. Consequences of inaccurate coding and billing in the order of importance
1. Prison
6. Denied claims
2. Exclusion
7. Trigger a review
3. Sanctions
8. Returned claims
4. Fines
9. Suspended claims
5. False Claims Act
10. Downcoding

Consequences of inaccurate coding are disastrous as shown in Table 4.

\section{EVALUATION AND MANAGEMENT SERVICES}

Evaluation and management services are a separate issue and a major component of fraud and abuse. These are described in detail elsewhere $(1,6-8)$.

\section{CORRECT CODING EDITS FOR INTERVENTIONAL TECHNIQUES IN PAIN MANAGEMENT}

Various commonly utilized interventional techniques in pain management are described here with comprehensive, component, and mutually exclusive codes. However, if there is a question about coding, its comprehensive nature, or mutual exclusion, physicians and other providers are urged to contact local Medicare carriers or third-party payors. The information reproduced here is derived from the National Correct Coding Primer, a Medicare Part B publication (2), which obtains its information from the National Technical Information Service (NIIS) - the branch of HCFA that is responsible for the distribution of reedited files of Correct Coding Edits that are sent to resellers for formatting and printing. The recently released version 6.1 of the National Correct Coding Initiative was discovered to contain 3,782 errors. The resellers, including the Medical Management Institute (MMI), Medicode, NTIS, and St. Anthony's, released version 6.1 with the errors, as sellers are not allowed to revise data found within the reedited files released by NTIS. As per coding and Medicare updates, a publication of the MMI (9), NTIS has stated that it will not release either its printed or reedited electronic files to customers or resellers. It also appears that St. Anthony's, Medicode, and NTIS have stated that they will not release a corrected version of version 6.1 of the CCI, only that the changes will be reflected in version 6.2, which will not be released until July 1, 2000. However, other publishers have sent the corrected update to version 6.1, including Part B News Group, Rockwell, Maryland, from which the information here has been obtained.

\section{CONCLUSION}

The HCFA, to help ensure that providers across various jurisdictions receive like payments for the same services, use the same codes and provide similar documentation for services performed, created the NCCC. Subsequently, HCFA established the National Correct Coding Policy in 
1996 and eventually implemented the "Medicare Correct Coding Initiative" to identify and isolate inappropriate coding, unbundling, and other irregularities in coding. Correct coding policies encompass coding based on standards of medical/surgical practice; medical/surgical packages; add-on codes; modifiers; excluded services; CPT procedure-code definitions; CPT coding manual instructions/guidelines; separate procedures; designation of sex; family of codes; most extensive procedures; sequential procedures; with or without procedures; laboratory panels; unlisted services or procedures; misuse of column II code with column I code; mutually exclusive procedures; and, finally, Correct Coding Edits modifier indicated. Interventional pain physicians, like the rest of the medical community, are deeply entangled in National Correct Coding Policy and CCI. It is imperative for interventional pain physicians to avoid incorrect coding, either by unbundling or upcoding, and to understand National Correct Coding Policy and Correct Coding Edits, some of which are described here.

\section{REFERENCES}

1. Manchikanti L. The impact of National Correct Coding Policy on interventional pain management. Pain Physician 1999; 2:33-45.

2. National Correct Coding Primer 2000: Part 'B', News Group, Rockville, MD.

3. CCI edits require expertise in the face of voluminous outpatient, coding changes. HCPCS Report 2000; 14:1-5.

4. Current Procedural Terminology. CPT 1999, Chicago, American Medical Association, 1998.

5. Current Procedural Terminology. CPT 2000, Chicago, American Medical Association, 1999.

6. Manchikanti L. Evaluation and management services in interventional pain practice: Doing it right! Pain Physician 2000; 3:322-341

7. Manchikanti L. The role of evaluation and management services in pain management. Pain Physician $1999 ; 2: 10-32$.

8. Manchikanti L. Appropriate documentation, billing and coding in interventional pain practice. Pain Physician 2000; 3:218-236.

9. CCI version 6.1 Errata. Coding and Medicare updates. 2000: May 1-2.

10. Manchikanti L. CPT 2000: Interventional pain management coding in the new millennium. Pain Physician 2000; 3:73-85. 